\title{
Effects of Organic Turmeric on Liver Integrity and Oxidative Stress of the Brain in Rabbits Exposed to Ultraviolet Radiation
}

\section{BAKI, Oladimeji Ibraheem ${ }^{1}$; OYEDUN, Ifeoluwa Oluwagbenga ${ }^{2}$; OWOLABI, Olamide Tawa ${ }^{3}$; OGUNSHOLA, Olawale Jacob² and ADISA, Babatunde} Ibrahim $^{1}$

\author{
${ }^{1}$ Department of Animal Production and Health, Ladoke Akintola University of Technology, Ogbomoso, Oyo State, Nigeria. \\ Email: oladimejibaki@yahoo.com \\ ${ }^{2}$ Department of Animal Production and Health, Federal University of Technology, Akure, Ondo State, Nigeria. \\ Email:feyisayogood0551@hotmail.com, ojogunshola@futa.edu.ng \\ ${ }^{3}$ Department of Animal Health and Production Technology, Rufus Giwa Polytechnic, Owo, Ondo State, Nigeria \\ Email:owolabiolamide98@gmail.com
}

\begin{abstract}
This project investigated the effects of organic turmeric on the liver and oxidative stress of the brain in rabbit acutely exposed to ultraviolet radiation. Thirty five weaned rabbits between 8-10 weeks of age, randomly allocated to control (A) and five (5) treatments: $B, C, D, E$ and $F$ were used for this experiment. Treatment A: fed organic feed without turmeric inclusion and not radiated, Treatment B: fed diet supplemented with $2 \%$ turmeric as its constituents but not radiated, Treatment $C$ : fed organic feed without turmeric inclusion before but after radiation, Treatment $D$ : fed organic feed without turmeric inclusion before and after radiation. Treatment E: fed diet supplemented with 2\% turmeric before but not after radiation. Treatment F: fed diet supplemented with $2 \%$ turmeric before and after radiation. There were significant $(p<0.05)$ differences in superoxide dismutase, catalase and melondialdehyde. Histological studies reveals that the treatments radiated showed structural differences from the control. Liver section in Treatment D showed portal triad infiltrated by lymphocytes with less vacuolar degeneration. The liver damage was mild in Treatments $C$ and $E$. The histology of rabbits in treatment $A$ and $F$ revealed normal liver hepatocytes and portal triad. It was concluded that ultraviolet $(U V)$ radiation resulted in oxidative stress in the brain and feeding of $2 \%$ organic turmeric supplemented diet before and after exposure to radiation seen to have be effective against oxidative damages caused by ultraviolet radiation. Also, UV radiation has detrimental effect on the liver and organic turmeric had hepatoprotective and antioxidant properties.
\end{abstract}

Keywords-Brain, Liver, Oxidative Stress, Organic Turmeric, Rabbits, Ultraviolet Radiation

\section{INTRODUCTION}

Rabbit (Oryctolagus cunniculus) is one of the animals that can be reared successfully at family level (FAO, 1996). It is characterized by short gestation interval and very good source of protein with balanced amino acid profile. Its meat is appreciated for its properties in particular through its high protein/energy ratio. It has high essential fatty acid content and low cholesterol (Xiccato, 1999). The daily weight gain is high in proportion to the body weight which gives them a rapid growth rate, and sexual maturity is early (Ajayi et al., 2005). The stratosphere ozone layer forms a thin shield in the upper atmosphere, protecting life on the earth from the sun's ultraviolet (UV) radiation. In the 1980s, scientist found evidence that the ozone layer was depleted (EPA, 2010). Depletion of ozone layer results in increased UV radiation reaching the earth's surface. UV is invisible and does not produce immediate reaction. UV radiation leads to the damage of cellular constituents, resulting in a complex cell response that includes induction of genes and perturbation of a variety of signaling pathways (Bender et al., 1997).

Oxygen is a highly reactive atom that is capable of becoming part of potential damaging molecules commonly called "free radicals". Free radicals are capable of attacking the healthy cells of the body, causing them to lose their structure and function. Cell damage caused by free radicals appears to be a major contributor to ageing and to degenerative diseases of 
ageing such as cancer, cardiovascular disease, cataracts, immune system decline, and brain dysfunction (Sies et al., 1999). Certain organs system in humans is susceptible to greater level of oxidative stress. The brain, which accounts for only $2 \%$ of the body weight, consumes $20 \%$ of the total oxygen inspired. The brain processes much $\mathrm{O}_{2}$ per unit tissue mass. The nervous system is rich in both unsaturated fatty acid and iron, the high lipid content of nervous tissue, coupled with its high aerobic metabolic activity makes it susceptible to oxidative alteration (Bauer and Bauer, 1999).

Liver is a vital organ present in vertebrates and some other animals. It has a wide range of functions, including detoxification, protein synthesis and production of biochemicals, necessary for digestion. Excessive iron deposition in the liver can lead to further injury such as hepatocellular necrosis, inflammation, fibrosis and in some cases even to carcinoma (Zhao et al., 2005). In recent years, several hundred plants have been examined for their use in a wide variety of liver disorders. These plants include Silybum marianum (milk thistle), Picrorhiza kurroa (kutkin), Camellia sinensis (green tea) and Glycyrrhiz aglabra and Curcuma longa (Turmeric) (Luper, 1999). Curcumin is the principle active ingredient in turmeric, a spice derived from the rhizome of Curcuma longa. Turmeric is used in both Chinese and Indian traditional medicines (Aggarwal et al., 2006), with applications as an anti-inflammatory agent, for peptic ulcer and dyspepsia, in skin diseases and wound healing and in liver and urinary tract diseases (Luper et al., 1999). Even very high level of curcumin and related curcuminoids had low toxicity at oral doses up to $12 \mathrm{~g}$ per day in humans (Lao et al., 2006). Curcumin is also effective in preventing chemically-induced liver damage and prevented carcinogenic effects of the hepatocarcinogens afflatoxin or nitrosodiethylamine (Sharma et al., 2005). It reduced liver fibrosis in a rat model of nonalcoholic steatohepatitis and in rats given thioacetamide (Leclercq et al., 2004).

\section{MATERIALS AND METHODS}

\subsection{Experimental Site}

This experiment was carried out at the rabbitry unit of the Teaching and Research farm, Ladoke Akintola University of Technology (LAUTECH) Ogbomoso. Ogbomoso is located in the derived savannah zone of Nigeria. It lies on longitude $4^{0}$ $15^{1}$ East greenwish meridian and latitude $8^{0} 15^{1}$ North at the equator. The altitude is between 300 and $600 \mathrm{~m}$ above sea level while the mean temperature and annual rainfall are $27^{\circ} \mathrm{C}$ and $1247 \mathrm{~mm}$ respectively

\subsection{Experimental Materials}

Three wooden cages with 24 hutches in each cage were used for this experiment. The dimension of the hutches was $51 \mathrm{~cm}$ length by $62 \mathrm{~cm}$ breath, large enough to contain 5 rabbits of age 8-10 weeks at a time. However, two rabbits were placed in each hutch. Thirty five (35) weaned rabbits of average weight $1200-1300 \mathrm{~g}$ purchased from reliable source were used for this experiment. The does and the bucks were separated, so as to prevent mating.

$200 \mathrm{~kg}$ of Turmeric rhizome was purchased from Euro bridge farm Odogbolu, Ogun State, and was separated from all forms of attached soil before being boiled and sliced to increase the surface area. The sliced samples were air dried to reduce moisture content. The dried turmeric was ground into powder with an electric grinder and sieved so as to have uniform size of powder.

Organic turmeric $\rightarrow$ boiling $\rightarrow$ slicing $\rightarrow$ drying $\rightarrow$ grinding Exposure to UV radiation was done by putting them in a wooden artificial radiation box. 3 florescence UV bulbs were used and exposure was done for ten minutes for 10consecutive days with 5 rabbits at a time.

\subsection{Feed Formulation and Feeding}

Two diets were formulated, turmeric was not included as one of the constituents of the diet 1 but was included in the diet 2 . Turmeric was added at $2 \%$ inclusion in the feed. Garlic, Asparagus racemosus and Moringa oleifera were included as premix to take care of ethnoveterinary requirement. The rabbits were fed ad-libitum. The gross composition of the experiment diets is presented in TABLE 1.

\subsection{Treatments}

Thirty five rabbits were allocated into a control and five treatment groups as follow: Control A: Eight rabbits, fed organic feed without turmeric inclusion and not radiated. Treatment B: Seven rabbits, fed diet supplemented with $2 \%$ turmeric as its constituents but not radiated. Treatment C: Five rabbits, fed organic feed without turmeric inclusion before but turmeric after radiation. Treatment D: Five rabbits, fed organic feed without turmeric inclusion before and after radiation. Treatment E: Five rabbits, fed diet supplemented with $2 \%$ turmeric before but not after radiation. Treatment F: Five rabbits, fed diet supplemented with $2 \%$ turmeric before and after radiation.

\subsection{Data Collection}

At the end of the third week of feeding after radiation, the rabbits were sacrificed through cervical dislocation. The brains were removed and apportion of each brain was homogenized with 3 -sucrose buffer solution of $0.25 \mathrm{mls}$ with $\mathrm{pH}$ of 7.4 in a mortar and a pestle. The homogenates were stored in ice-block and later processed for oxidative stress determination with three different oxidative stress markers.

\subsection{Assay Methods}

2.6.1 Determination of Lipid Peroxidation (MDA) 
Principles: Assessment of lipid peroxidation was carried out based on the principle of Varshney and Kale (1990). Estimation of lipid peroxidation was based on the reaction of melondialdehyde with thiobarbituric acid (TBA) forming a MDA-TEAR adduct that absorb light strongly at $532 \mathrm{~mm}$.

Procedure: $0.4 \mathrm{ml}$ of reaction mixture i.e. sample already quenched with $0.5 \mathrm{ml}$ of $30 \%$. TCA was added to $1.6 \mathrm{ml}$ of Trihydroxymethyl methylamine potassium chloride at PH 7.4. $0.5 \mathrm{ml}$ of $8 \%$ TBA was added and incubated for 45 minutes at $300 \mathrm{C}$ to produce a pink coloured reaction mixture which was centrifuge at $1400 \mathrm{rpm}$ for 15 minutes. The absorb area of the clear supernatant was then read at $532 \mathrm{~nm}$.

MDA (units) $=$ Absorbance $X$ Volume of Mixture E532 X Volume of Sample

\subsubsection{Determination of Superoxide Dismutase (SOD)} Activity

SOD activity was determined by the method described by Misra and Fridorich (1972)

Principle: the ability of superoxide dismutase to inhibit the auto oxidation of epinephrine at PH 10.2 to adrenochrome makes this reaction a basis for a simple assay of dismutase. Procedure: $1 \mathrm{ml}$ of sample was diluted in $9 \mathrm{ml}$ of distilled water to make a 1 in 10 dilutions. An aliquot of the diluted sample was added to $25 \mathrm{ml}$ of $0.05 \mathrm{M}$ carbonate buffer $\mathrm{pH} 10.2$ to equilibrate in the spectrophotometer and the reaction was initiated by adding $0.3 \mathrm{ml}$ of adrenaline. The change in absorbance was monitored at $430 \mathrm{~nm}$ for 5 minutes.

\subsubsection{Determination of Catalase (CAT) Activity}

Catalase activity was determined according to the method described by Aebi (1983).

\subsection{Statistical Analysis}

All data were subjected to Analysis of Variance (ANOVA) using SAS (2008). Duncan Multiple Range Test of the same
Principle: Catalase catalyses the decomposition of hydrogen peroxide $\left(\mathrm{H}_{2} \mathrm{O}_{2}\right)$ to water and oxygen. Hydrogen peroxide is formed in the eukaryotic cells as a by-product of various oxidase and seperoxide reactions. Hydrogen peroxide is highly deleterious to the cells and its accumulation causes oxidation of cellular targets such as DNA, protein and lipid leading to mutagenesis and cell death. Removal of the $\mathrm{H}_{2} \mathrm{O}_{2}$ from the cell by catalase provides protection against oxidative damage to the cell.

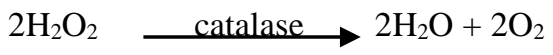

Procedure: $0.1 \mathrm{ml}$ of the sample was pipette into curette containing $1.9 \mathrm{ml}$ of $50 \mathrm{MM}$ phosphate buffer $\mathrm{PH} 7.0$. The reaction was initiated by addition of $0.1 \mathrm{ml}$ of freshly prepared $30 \%(\mathrm{w} / \mathrm{v})$ hydrogen peroxide. The rate of decomposition of hydrogen peroxide was measured spectrophotometer at $240 \mathrm{~nm}$

\subsection{Histology}

The livers of 35 matured rabbits ( 25 males and 10 females) were used for this study after which they were sacrificed via cervical dislocation at the Animal Production and Health Laboratory (LAUTECH). The eviscerated liver of the rabbits was weighed and transferred into containers containing formal saline fixative. The livers were dehydrated through varying concentration of alcohol and cleared with xylene. The tissues were subsequently impregnated and embedded in paraffin wax. They were then cut at 3-5 microns, dewaxed and stained with haematoxylin and eosin. The slides were reviewed and photomicrographs taken with a digital camera attached to a light microscope.

statistical package was used to separate the mean among treatment. 
Table.1: Feed Formulation Table

\begin{tabular}{lll}
\hline Ingredients(kg) & Diet 1(No Turmeric) \% & Diet 2 (Turmeric) \% \\
\hline Maize & 18 & 18 \\
Maize bran & 10.8 & 8.8 \\
Wheat offal & 12 & 12 \\
P.K.C & 45 & 45 \\
Fish meal 72\% & 2 & 2 \\
Bone meal & 2 & 2 \\
Oyster shell & 1.5 & 1.5 \\
Salt & 0.25 & 0.25 \\
Moringa oleifera & 0.2 & 0.2 \\
Ginger & 0.09 & 0.09 \\
Garlic & 0.08 & 0.08 \\
Asparagus racemosus & 0.08 & 0.08 \\
Tumeric & - & 2 \\
Total & 100 & 100 \\
Calculated Proximate Composition of Diet & \\
Energy & & $2360.07 \mathrm{ME} \mathrm{cal} / \mathrm{kg}$ \\
Crude protein & & $18.17 \%$ \\
Ether extract & & $4.75 \%$ \\
Lysine & & $0.68 \%$ \\
Methionine & & $0.33 \%$ \\
Crude fibre & & $8.46 \%$ \\
Calcium & & $1.44 \%$ \\
Phosphorus & & $0.49 \%$ \\
\hline
\end{tabular}

Table.2: Level of Oxidative Stress in the Brain of Male Rabbits by Different Markers

\begin{tabular}{llll}
\hline Treatment & SOD $(\mathbf{n m o l} / \mathbf{L})$ & CAT $(\mathbf{n m o l} / \mathbf{L})$ & MDA $(\mathbf{n m o l} / \mathbf{L})$ \\
\hline $\mathrm{A}$ & $22.36 \pm 1.08^{\mathrm{a}}$ & $14.21 \pm 3.18^{\mathrm{a}}$ & $41.02 \pm 2.72^{\mathrm{a}}$ \\
$\mathrm{B}$ & $16.52 \pm 0.69^{\mathrm{c}}$ & $14.95 \pm 1.49^{\mathrm{b}}$ & $32.14 \pm 6.16^{\mathrm{b}}$ \\
$\mathrm{C}$ & $16.98 \pm 1.16^{\mathrm{c}}$ & $14.40 \pm 1.61^{\mathrm{a}}$ & $16.44 \pm 1.05^{\mathrm{d}}$ \\
$\mathrm{D}$ & $15.50 \pm 1.25^{\mathrm{b}}$ & $11.50 \pm 1.32^{\mathrm{a}}$ & $26.15 \pm 1.50^{\mathrm{a}}$ \\
$\mathrm{E}$ & $16.90 \pm 1.66^{\mathrm{c}}$ & $13.21 \pm 1.39^{\mathrm{a}}$ & $30.30 \pm 2.32^{\mathrm{b}}$ \\
$\mathrm{F}$ & $22.32 \pm 0.72^{\mathrm{c}}$ & $15.30 \pm 1.10^{\mathrm{b}}$ & $42.50 \pm 2.42^{\mathrm{c}}$ \\
\hline$a, b, c=$ means on the same column but with different superscripts are statistically $(p<0.05)$ significant. SOD $=$ Superoxide
\end{tabular}

Dismutase CAT = Catalase $; M D A=$ Malondialdehyde $; \mathrm{A}=$ fed organic feed without turmeric inclusion and not radiated; $\mathrm{B}=$ fed diet supplemented with $2 \%$ turmeric as its constituents but not radiated; $\mathrm{C}=$ fed organic feed without turmeric inclusion before but turmeric after radiation; $\mathrm{D}=$ fed organic feed without turmeric inclusion before and after radiation; $\mathrm{E}=$ fed diet supplemented with $2 \%$ turmeric before but not after radiation; $\mathrm{F}=$ fed diet supplemented with $2 \%$ turmeric before and after radiation

\section{RESULTS}

\subsection{Oxidative Stress of the Brain}

Level of oxidative stress in the rain is showed in TABLE 2. There were significant $(\mathrm{p}<0.05)$ differences in superoxide dismutase (SOD) in which treatment A (those fed organic feed without turmeric and not radiated) had the highest $(22.36 \pm 1.08 \mathrm{nmol} / \mathrm{L})$ value while treatment $\mathrm{D}$ (those fed organic feed without turmeric before and after radiation) had the least $(15.50 \pm 1.25 \mathrm{nmol} / \mathrm{L})$ value of SOD. The significant differences observed in catalase indicated that rabbit fed organic feed with turmeric before and after radiation (Treatment F) appears to have the highest $(15.30 \pm 1.10 \mathrm{nmol} / \mathrm{L})$ values while the least $(11.50 \pm 1.32 \mathrm{nmol} / \mathrm{L})$ was recorded for Treatment $\mathrm{D}$ (fed organic feed without turmeric before and after radiation). There were significant $(\mathrm{p}<0.05)$ observed in melondialdehyde (MDA), highest $(42.50 \pm 2.42 \mathrm{nmol} / \mathrm{L})$ and the least $(16.44 \pm 1.05 \mathrm{nmol} / \mathrm{L})$ values of MDA were recorded in treatment $\mathrm{F}$ (those fed organic feed with turmeric before and after radiation) and $\mathrm{C}$ (those fed diet supplemented with $2 \%$ turmeric after radiation) respectively. 


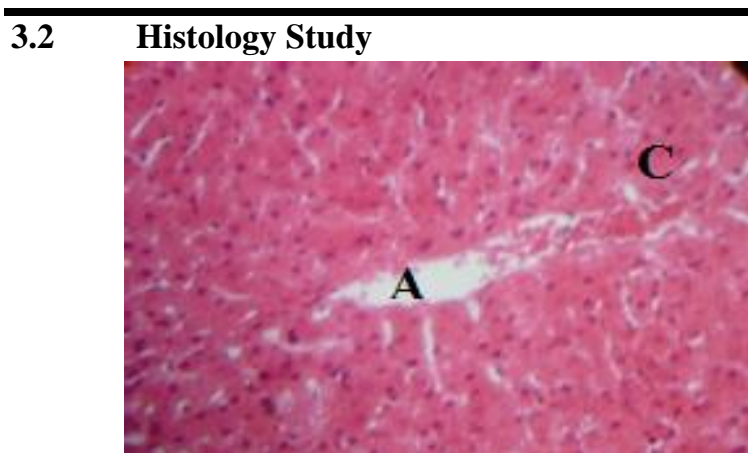

Fig. 1: Photomicrograph of Liver from rabbit fed organic feed without turmeric inclusion and not radiated (Control A) showing normal liver architecture; normal hepatocyte $(C)$ and portal vein (A) (Mag $x$ 400).

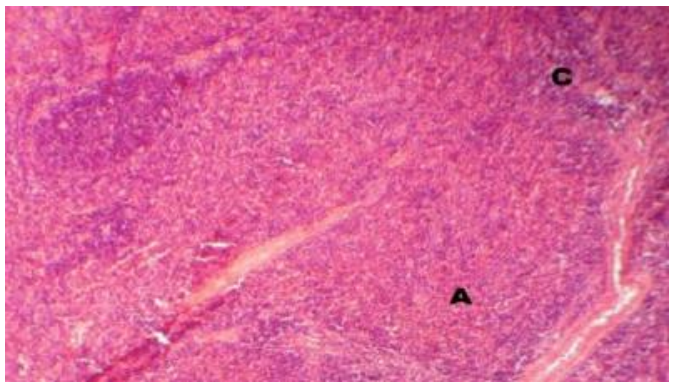

Fig.2: Photomicrograph of Liver from rabbits fed organic feed with inclusion of turmeric throughout and they are not radiated (Treatment $B$ ). It shows mild vacuolar degeneration

(A) with focal necrosis of hepatocyte especially around central veins (C) (Mag x 100), which may due to environmental factors.

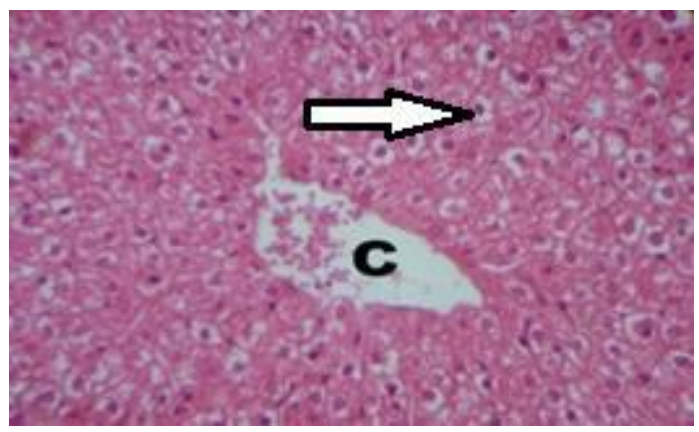

Fig.3: Photomicrograph of Liver from rabbits fed organic feed without turmeric inclusion before but after radiation

(Treatment C). It shows normal hepatic cells each with welldefined cytoplasm, prominent nucleus, nucleolus (Arrow) and well brought out central vein (C) (Mag $x$ 400).

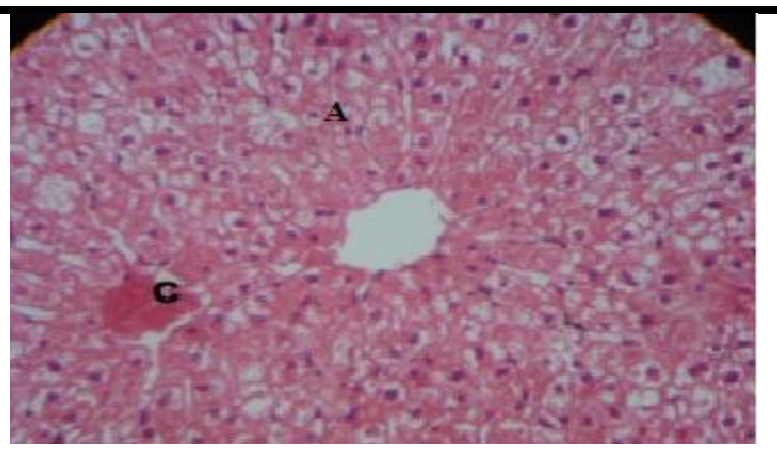

Fig.4: Photomicrograph of Liver from rabbits fed organic feed without inclusion of turmeric before and after radiation

(Treatment D) showing portal triad infiltrated by lymphocytes $(C)$ with less vacuolar degeneration (A) (Mag $x$ 400).

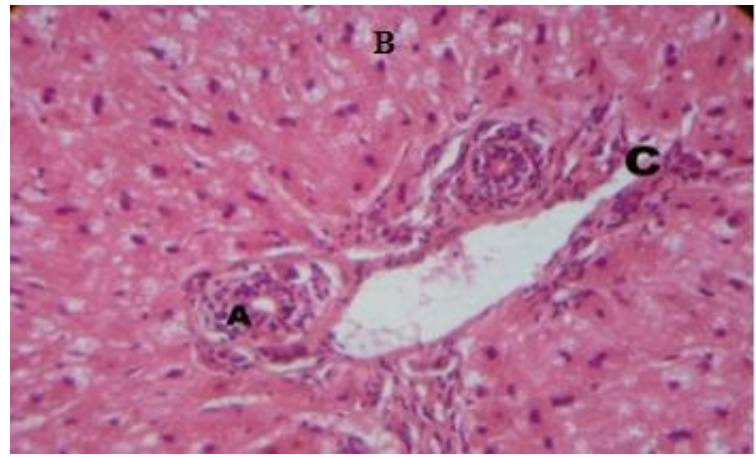

Fig.5: Photomicrograph of Liver from rabbits fed turmeric supplemented diet before radiation but no turmeric after radiation (Treatment $E$ ) showing diffuse mild vacuolar degeneration $(B)$, mild inflammation of portal triad $(A)$ and normal venule (C) (Mag $x$ 400).

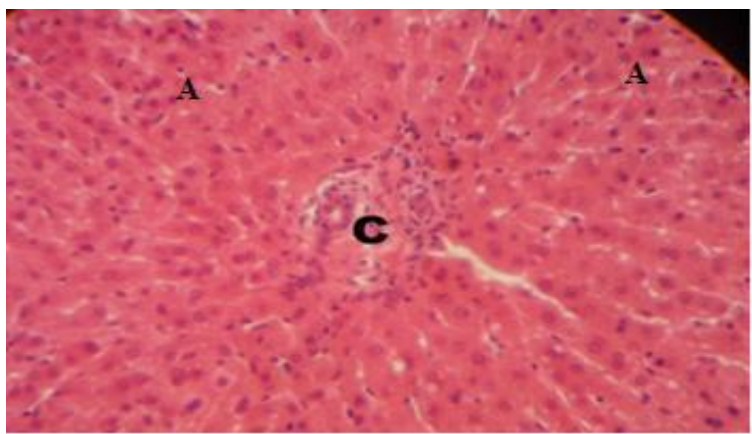

Fig.6: Photomicrograph of Liver from rabbits fed organic turmeric supplemented diet before and after radiation

(Treatment $F$ ) showing normal hepatocytes (black arrow) and portal triad (C) (Mag $x$ 400).This confirm the hepatoprotective role of curcumin. 


\section{DISCUSSION}

Free radical have been implicated in many disease processes including cancer, while superoxide dismutase (SODs) are the main enzymes responsible for the elimination of superoxide radicals and are considered to be key antioxidants in aerobic cells (Hileman et al., 2001). The observed value for the treatment D (fed organic feed without turmeric before and after radiation) indicated that SOD and catalase revealed oxidative stress in the brain of pubertal rabbits exposed to ultraviolet radiation when compared with treatment $\mathrm{A}$. This implied that ultraviolet radiation exposed to, reduced the activities of antioxidant enzymes. Nagai et al. (2008) reported that superoxide dismutase catalyses the dismutation of superoxide into oxygen and hydrogen peroxide. Similar report was given by Chelikani et al. (2004) who reported catalase catalyses the decomposition of hydrogen peroxide to water and oxygen. Therefore, reduction in the number of these antioxidants in the brain indicates damaging effect of ultraviolet radiation. The significant differences observed in treatment $\mathrm{F}$ (rabbits fed organic feed with organic turmeric before and after radiation) for SOD and catalase indicated that turmeric fed before and after radiation ameliorates the effect of ultraviolet radiation. Similar reports were given by Chattopadhyay et al. (2004) who indicated that curcuminoids can reduce free radical compounds such as hydroxyl radicals and superoxide radicals in biological system. Melondialdehyde, one of the several molecular weight end products formed via decomposition of certain primary and secondary lipid peroxidation products (Janero and Burghardt, 1988). Melondialdehyde (MDA) revealed oxidative stress in the brain of pubertal rabbits exposed to radiation and fed turmeric supplemented diet after (treatment $\mathrm{C}$ ). This indicated that turmeric fed after radiation could not effectively prevent damages caused by ultraviolet radiation. High level of MDA observed in treatment $\mathrm{F}$ indicated that turmeric had prophylactic and therapeutic effects against ultraviolet radiation.

Histology revealed that the treatments that were radiated have structural differences compared to the control. Liver section of rabbit, control (treatment A) as seen in Fig. 1 exhibited normal hepatic cells, each with well-defined cytoplasm, prominent nucleus and nucleolus and well brought out central vein. These features gave an indication of normal hepatic architectural integrity. In treatment B, rabbits were fed organic turmeric supplemented diet but were not radiated. Liver section shows mild vacuolar degeneration with focal necrosis of hepatocyte especially around central veins as displayed in Fig. 2. Some rabbits in this group may susceptible to disease due to environmental factors.
Histology of the Rabbits that fed organic feed without turmeric inclusion before but turmeric after radiation as pictured in Fig. 3 shows normal hepatocytes around portal triad with welldefined cytoplasm, prominent nucleus and nucleolus. This confirmed the hepatoprotective role of turmeric. Similar reports were given by Singh et al. (2011). Liver histology of rabbits, fed organic feed without inclusion of turmeric before and after radiation (Fig. 4) shows portal triad infiltrated by lymphocytes with less vacuolar degeneration. The damage caused by ultraviolet radiation is more severed because TLymphocyte is the main mononuclear cell infiltrating portal triad as in primary biliary cirrhosis (Whiteside et al., 1984). The histology of the liver of the rabbit in this treatment confirms that ultraviolet radiation may be responsible for the liver damage (Zhao et al., 2005).Histology of rabbits fed organic feed with inclusion of $2 \%$ turmeric before radiation but no turmeric after radiation i.e. treatment E (Fig. 5) and those fed turmeric supplemented diet before and after radiation i.e. treatment $\mathrm{F}$ (Fig. 6) shows diffuse mild vacuolar degeneration and normal hepatocytes and portal triad respectively. The effect of the ailment on the liver is mild and not significant in treatment $\mathrm{E}$. This means that the turmeric that was fed to the rabbits before radiation in treatment $\mathrm{E}$ ameliorate the damages caused by radiation on the liver. When compared the histology of treatment D (Fig. 4) to that of treatment C (Fig. 3); it was observed that the damages caused by radiation on the liver in rabbits of treatment D (Fig. 4) was more pronounced compare to that of treatment $\mathrm{C}$ (Fig. 3). Treatment F (Fig. 6) shows normal hepatocytes and normal portal triad which is similar to control while treatment $\mathrm{E}$ shows diffuse mild vacuolar degeneration. This finding correlates with the work of Kuttan (1985) who reported the use of curcumin in wound healing, liver ailments, hepatitis, urinary tract disease and as a cosmetic compound. Also, Feroz and Nahida (2013) reported that roots of Paeonia officinalis Linn serve as hepatoprotective. Therefore, the hepatoprotective and antioxidant role of turmeric on the liver was confirmed.

\section{CONCLUSION}

It was concluded that Ultraviolet (UV) radiation resulted in oxidative stress in the brain which could be detrimental to the brain function. Feeding of $2 \%$ organic turmeric supplemented diet before and after exposure to radiation seen to have be effective against oxidative damages caused by ultraviolet radiation. Ultraviolet radiation caused a damaging effect on the liver through vacuolar degeneration and portal triad infiltration by lymphocytes of radiated rabbits and organic turmeric has hepatoprotective and antioxidant effects. 


\section{ACKNOWLEDGEMENT}

The Authors hereby express their profound gratitude to Late Prof. V. A. Togun for his fatherly advice, financial and technical support for this research. May his gentle soul rest in peace.

\section{REFERENCES}

[1] Aebi, H. (1983). Catalase. In methods of Enzymatic Analysis, Bergmeyer, H. Ed., Verlag, Chemie, Weinheim, 3:273-277.

[2] Aggarwal, B. B. \& Shishodia, S. (2006). Molecular Targets of Dietary Agents for Prevention and Therapy of Cancer. Biochemistry Pharmacology, 71(10):13971421.

[3] Bender, K., Blattner, C., Knebel, A., Iordanov, M., Herrlich, P. and Rahmsdorf, H. J. (1997). Uv-Induced Signal Transduction. Journal of Photochemistry and Photobiology, 37:1-17.

[4] Chattopadhuay, I., Biswas, K., Bandyopadhyay, U. and Ranajit .K (2004). Turmeric and Curcumin: Biological Actions and Medical Applications. Current Science, 87 (1): 44-53

[5] Chelikani, P., Fita, I. and Loewen, P C. (2004). Diversity of Structures and Properties among Catalase. Cell Molecular of Life Science, 61(2): 192-208

[6] EPA (2010). Stratospheric Ozone Layer Depletion. Air and Radiation. Journal of Environmental Protection Agency, 43:10-22.

[7] FAO (1985). Expert Committee Report on Agriculture, Food and Agriculture, Rome.

[8] Feroz, A. and Nahida, T. (2013). Preliminary Phytochemical, Acute Oral Toxicity and Antihepatotoxic Study of Roots of Paeonia officinalis Linn. Asian Pacific Journal of Tropical Biomedicine, 3(1): 64-68.

[9] Hileman, E. A., Achanta, G. and Huang, P. (2001). Superoxide Dismutase: An Emerging Target for Cancer Therapeutics. Expert Opin. Ther. Targets, 5: 697-710.

[10] Janero, D. R. and Burghardt, B. (1988). Analysis of Cardiac Membrane Phospholipid Peroxidation Kinetics As Melondialdehyde: Non-Specificity of Thiobarbuturic Acid-Reactivity. Lipids, 23(5): 452-458

[11] Kuttan, R. (1985). Potential Anticancer Activity of Turmeric (Curcuma Longa).Cancer Letter, 29:197-202.

[12]Luper, S. (1999). A Review of Plants Used In the Treatment of Liver Disease: Part Two. Alternative Medical Revolution, 4(3):178-188.

[13] Misra, H. P. and Fridovich, I. (1972). The Role of Superoxide Anion in the Autoxidation of Epinephrine and a Simple Assay for Superoxide Dismutase, The Journal of Biological Chemistry, 247:3170-3175

[14] Nagai, R., Fajuwa, Y. and Mera, K. (2006). Usefulness of Antibodies for Evaluating the Biology Significance of Age. Annual New York Academic Science, 1126: 38-41

[15] Singh, H., Bedi, P. S. and Singh, B. (2011). Hepatoprotective Activity of Turmeric And Garlic Against 7-12, Dimethylbenzanthracene Induced Liver Damage in Wistar Albino Rats. European Journal of Medicinal Plants, 1(4): 162-170

[16] Varshney, R. and Kale, R. K. (1990). Effects of Calmodulin Antagonists on Radiation-induced Lipid Peroxidation in Microsomes. Int. J. Rad. Biol., 58:733743

[17] Whiteside, T. L., Schade, R. R., Starzl, T. E. And Vanthiel, D. H. (1984). Mononuclear Cell Infiltrating Portal Triad;Jully,4(4): 262-72

[18]Xiccato, G. (1999). Feeding and Meat Quality in Rabbits: A Review 1. World Rabbit Science, 7(2): 75-86.

[19]Zhao, Y., Li, H., Gao, Z. and Xu, H. (2005). Effects of Dietary Baicalin Supplementation on Iron OverloadInduced Mouse Liver Oxidative Injury. European Journal of Pharmacology, 509(2-3): 195-200. 